\title{
¿PUEDE EL OPTIMISMO MEDIAR EL EFECTO NEGATIVO DE LA ANSIEDAD RASGO SOBRE EL BIENESTAR PSICOLÓGICO?
}

\author{
Andrés Gutiérrez-Carmona a \& b1 ${ }^{\mathbb{D}}$, Carolina Alday Mondaca ${ }^{\mathfrak{D}}$, \\ Alfonso Urzúa ${ }^{\mathrm{a}}{ }^{\mathbb{D}}, \boldsymbol{\&}$ Anna Wlodarczyk ${ }^{\mathrm{a}}$ \\ Universidad Católica del Norte, Antofagasta, Chile. ${ }^{\mathrm{a}}$ \\ Universidad de Antofagasta, Antofagasta, Chile. ${ }^{\mathrm{b}}$
}

\section{RESUMEN}

El objetivo del estudio fue evaluar el efecto mediador del optimismo en la relación ansiedad-rasgo y el bienestar psicológico en estudiantes de educación superior. La muestra estuvo constituida por 332 estudiantes de educación superior de Antofagasta, Chile, con una media de edad de 21.8 años. Se encuentra que el efecto negativo de la ansiedad-rasgo sobre las dimensiones de bienestar psicológico disminuye al incorporar al optimismo en la regresión. Se concluye que el optimismo tiene un efecto de mediación parcial sobre las dimensiones del bienestar psicológico, salvo en crecimiento personal, donde ejerce una mediación total.

\section{Palabras Claves}

bienestar psicológico; ansiedad-rasgo; optimismo; estudiantes universitarios; educación superior

\begin{abstract}
The objective of the study was to evaluate the mediating effect of optimism in the anxiety-trait relationship and psychological well-being in higher education students. The sample consisted of 332 students of higher education in Antofagasta, Chile, with an average age of 21.8 years. It is found that the negative effect of anxiety-trait on the dimensions of psychological well-being diminishes when incorporating optimism in the regression. It is concluded that optimism has a partial mediating effect on the dimensions of psychological well-being, except in personal growth, where it exercises total mediation.
\end{abstract}

Keywords

psychological well-being; anxiety-trait; optimism; university students; higher education

\footnotetext{
${ }^{1}$ Correspondence about this article should be addressed to Andrés Gutiérrez Carmona, Avenida Angamos 0610, Antofagasta, Chile. E-mail: andres.gutiérrez.carmona@uantof.cl
} 


\section{CAN OPTIMISM MEDIATE THE NEGATIVE EFFECT OF TRAIT ANXIETY ON PSYCHOLOGICAL WELL-BEING?}

\section{Introducción}

Uno de los grandes desafíos de la educación universitaria actual, es el de formar individuos competentes, autónomos, capaces de comunicarse en forma efectiva y de adaptarse a diferentes contextos en una sociedad cada vez más globalizada (Villarroel \& Bruna, 2014). Esta línea de formación se vincula directamente con el desarrollo y mantención del bienestar psicológico en los estudiantes de educación superior, frente a lo cual, ya algunos autores, recomiendan la instalación de iniciativas que promuevan el desarrollo de los recursos y las fortalezas personales de los estudiantes a través del desarrollo de competencias genéricas actitudinales en el pregrado (Freire, Ferradás, Núñez, \& Valle, 2017).

La investigación actual sobre bienestar se ha derivado de dos perspectivas generales: el enfoque hedónico (bienestar subjetivo), el cual se centra en la felicidad y define el bienestar en función de alcanzar el placer y la evitación al dolor, y el enfoque eudaimónico (bienestar psicológico) el cual se centra en el desarrollo humano y su autorrealización, y define bienestar en la medida en que un ser humano se sienta en plenitud (Ryan \& Deci, 2001).

El bienestar psicológico (BP) o eudaimónico surge en base a concepciones del bienestar relacionadas con las cualidades de un ser humano maduro (Allport, 1961), la autorrealización del ser humano (Maslow, 1968) y el funcionamiento pleno de este (Rogers, 1961). Carol Ryff (1989) articula estas concepciones y define BP como el desarrollo del verdadero potencial de uno mismo. De esta manera, las expectativas, los ideales y las realidades perseguidas o conseguidas por las personas, expresadas en competencias personales, determinarían el bienestar psicológico, el cual también está influenciado, por su capacidad para afrontar las diversas situaciones vitales (Molina \& Meléndez, 2006).

Se ha reportado que el cursar una carrera universitaria, el deseo de perfeccionarse, la sensación de crecimiento y la percepción de estar desarrollando potencialidades, pueden influir positivamente en el nivel de bienestar psicológico general de los estudiantes (Freire, 2014; Villaseñor-Ponce, 2010). No obstante, se debe considerar que el bienestar psicológico está determinado también, por factores sociales y personales del individuo. Diferentes estudios han demostrado como el contexto sociocultural influye en 
el nivel bienestar y en el significado social e individual de "estar bien", "sentirse bien" o "tener bienestar" (Ryff, 1989; Schimmack, Radhakrishnan, Oishi, Dzokoto, \& Ahadi, 2002; Triandis, 1994; D’Anello, 2006, Muratori \& Bobowik, 2015). En este sentido, se ha visto, como el contexto disciplinar y académico puede estar relacionado con el nivel de bienestar psicológico de los estudiantes de diferentes carreras (Veliz-Burgos \& Apodaca, 2012), y como el nivel BP general varía en función del año académico que esté cursando el estudiante, no demostrándose si, una línea clara de variación (Sandoval, Dorner \& Véliz, 2017).

En cuanto a las variables personales, Ryff (1989) señala que el BP varía según el sexo y la edad de las personas, fundamentando que, al acercarse a la adultez, como en el caso de los estudiantes universitarios, se fortalecen las dimensiones del BP de autonomía, dominio del entorno y crecimiento personal. En cuanto a la relación sexo y BP, los estudios recientes no han sido concluyentes, pues en algunos se han encontrado relaciones significativas entre sexo femenino y mayor nivel de bienestar psicológico (Velásquez et. al., 2008; Zubieta, Muratori, \& Fernández, 2012; Perez, 2012; García-Alandete, 2012), en tanto en otros, no se han registrado diferencias significativas (Casullo, 2002; Chávez, 2006).

Los estudios han demostrado que los rasgos de personalidad están estrechamente relacionados con el $\mathrm{BP}$, tanto desde una perspectiva emocional, como cognitiva (Carmona-Halty \& Rojas-Paz, 2014; Weiss, Bates, \& Luciano, 2008), encontrándose una amplia evidencia científica que asocia fuertemente al BP con variables como la extraversión, el neuroticismo y la responsabilidad (DeNeve \& Cooper, 1998; Grant, Langan-Fox, \& Anglim, 2009; Schmutte \& Ryff , 1997; Steel, Schmidt \& Shultz, 2008).

Otra variable personal que ha demostrado estar relacionada con el BP es la ansiedad (Gutiérrez, 2017; Pardo, 2010; Ruini et al., 2003; Shek, 1993; Taoka et al., 2014; Villaseñor-Ponce, 2010), la cual además, se ha estudiado ampliamente en estudiantes universitarios, debido a que influye negativamente en el proceso de aprendizaje (Tirado, 2005; Villaseñor-Ponce, 2010), y a que sus síntomas, de alta prevalencia entre los estudiantes universitarios (Bodas, Ollendick y Sovani, 2008; Cardona-Arias, PérezRestrepo, Rivera-Ocampo, Gómez-Martínez, \& Reyes, 2015; Agudelo, Casadiegos \& Sánchez, 2008), repercuten en forma negativa en la vida social, en el desarrollo de habilidades sociales y en el rendimiento académico de los estudiantes (Tirado, 2005; Agudelo, Casadiegos, \& Sánchez, 2008; Villaseñor-Ponce, 2010; Eum \& Rice 2011; Uras, Delle Poggi, Rocco, \& Tabolli, 2012). 
Spielberger (1989) distingue dos tipos de ansiedad: la ansiedad-estado (AE), que es un "estado emocional" inmediato y modificable en el tiempo, y la ansiedad-rasgo (AR) relacionada con características individuales de personalidad, y por lo tanto relativamente estables en el tiempo. Según Spielberger (1989), las personas con alto grado de ansiedadrasgo perciben un mayor rango de situaciones como amenazantes, lo que las predispone a sufrir ansiedad-estado más frecuentemente o con mayor intensidad.

La evidencia indica que tanto la AR como la AE se relacionan en forma negativa con el BP (Gutiérrez, 2017; Taoka et al., 2014; Shek, 1993; Villaseñor-Ponce, 2010) y con cada una de las dimensiones de este constructo (Pardo, 2010; Ruini et al., 2003). En el estudio de Pardo (2010), todas las dimensiones del BP, con excepción del crecimiento personal, obtuvieron una mayor correlación con la AR que con la AE, concluyendo que el incremento de estados momentáneos de ansiedad no se asocia a una disminución del $\mathrm{BP}$, en la magnitud que lo hacen los rasgos estables de ansiedad.

En contraste a los efectos de la ansiedad, la variable optimismo se ha correlacionado en forma positiva con el BP (Augusto-Landa, Pulido-Martos, \& LopezZafra, 2011; Ferguson \& Goodwin, 2010; Vera-Villarroel, Códova-Rubio \& CelisAtenas, 2009), proponiéndose incluso un modelo explicativo en donde el optimismo en una variable predisponente del bienestar (Vera-Villarroel, Pavez, \& Silva, 2012). Se ha visto también, que esta variable tiene una relación negativa con la ansiedad y otros efectos negativos (Abdel-Khalek, 2006; Brydon, Walker, Wawrzyniak, Chart, \& Steptoc, 2009; De Moor, et al., 2006; Kivimaki, et al., 2005; Pavez, Mena, \& Vera-Villarroel, 2012; Vera, 2006), constituyéndose en un factor protector contra la AR (Pavez, et al., 2012).

Dado que los síntomas ansiosos presentan una alta prevalencia en los estudiantes universitarios, y que niveles altos de ansiedad se correlacionan de forma negativa con el BP y sus dimensiones, vemos que es necesario el estudiar una variable que pueda mediar esta relación, surgiendo así nuestra pregunta de investigación: ¿Puede el optimismo mediar el efecto negativo de la AR sobre las diferentes dimensiones del BP?

La hipótesis del presente trabajo sostiene que el efecto negativo de la AR sobre las dimensiones del BP, es disminuido al mediar este efecto la variable OP. 


\section{Método}

\section{Diseño y tipo de estudio}

Estudio cuantitativo, diseño no experimental transversal correlacional.

\section{Participantes}

La muestra está compuesta por 332 estudiantes de educación superior, de los cuales el $70,2 \%$ son sexo femenino y $29,8 \%$ del sexo masculino, con un promedio de edad de 21,9 años ( $D S=2,6$ años). Los participantes fueron reclutados a través de un muestreo de tipo no probabilístico intencionado. Como criterio de inclusión se consideró a estudiantes mayores de 18 años, en situación de alumno regular en alguna de las instituciones de educación superior de la ciudad de Antofagasta, que accedieran a participar voluntariamente previa firma de un consentimiento informado.

\section{Instrumentos}

\section{Escala de Bienestar Psicológico de Ryff}

Adaptada por Van Dierendonck (2004) y traducida al español por Díaz, et al., (2006). Es un cuestionario de autoreporte de formato Likert que permite evaluar los distintos dominios del bienestar psicológico. Está compuesta por 29 ítems que se subdividen en 6 subescalas: propósito en la vida (objetivos vitales que permitan dar sentido a la vida), dominio del entorno (habilidad personal para elegir o crear entornos favorables para satisfacer los deseos y necesidades propias), crecimiento personal (empeño por desarrollar las potencialidades y seguir creciendo como persona), relaciones positivas con otras personas (mantenimiento de relaciones), autoaceptación (sentirse bien consigo mismo, actitudes positivas hacia uno mismo) y autonomía (capacidad para mantener las convicciones de uno mismo con autodeterminación) (Ryff \& Keyes, 1995; Ryff \& Singer, 2006 ). Su formato de respuesta es tipo Likert que se puntúa de 1 a 6 , donde 1 es igual a "totalmente en desacuerdo" y 6, a "totalmente de acuerdo". Para la interpretación, obtener mayores puntajes en las subescalas indica mayor bienestar psicológico. Se pide a la persona que marque el número que mejor represente su opinión, respecto de cómo se siente con su vida. 
Este instrumento ha mostrado tener buenos índices de fiabilidad y validez en la mayoría de las subescalas tanto en su versión española propuesta por van Dierendonck (2004) como su validación en población chilena (Chitgian-Urzúa, Urzúa, \& VeraVillarroel, 2013; Vera-Villarroel, Urzúa, Silva, Pavez, \& Celis-Atenas, 2013) у específicamente universitaria (Véliz, 2012). En el presente estudio se aplicó el Alfa de Cronbach para evaluar la fiabilidad de las escalas correspondientes a cada una de las dimensiones del bienestar psicológico, obteniéndose los siguientes resultados: Autoaceptación .782; Relaciones positivas .787; Autonomía .630; Dominio del entorno .637; Crecimiento personal .734; Propósito en la vida .824.

\section{Inventario Ansiedad rasgo-estado}

Se utilizó la escala de Ansiedad Estado - Rasgo (Spielberger et al., 1970). Este instrumento evalúa la Ansiedad Estado (A-E) (condición emocional transitoria de la persona), y en su modo Ansiedad Rasgo (A-R) entendida como una condición emocional permanente de tensión (Spielberger et al., 1970), dando cuenta de una característica relativamente permanente de la persona, relacionada con la tendencia a responder con un elevado grado de ansiedad frente a situaciones percibidas como amenazantes (Spielberger \& Díaz Guerrero, 1975; Villaseñor-Ponce, 2010). La adaptación en población chilena mostró una consistencia interna de .92 para la escala de A-E y de .87 para A-R (VeraVillarroel, et al., 2007).

Para los análisis de este estudio solo se utilizó la variable ansiedad-rasgo, evaluando su efecto sobre las diferentes dimensiones del BP, en mediación de la variable optimismo. En el presente estudio la escala de AR obtuvo un Alfa de Cronbach de .888.

\section{Cuestionario de Orientación Vital.}

Cuestionario de Orientación Vital Revisado (LOT-R) (Scheier, Carver \& Bridges, 1994) Este cuestionario está compuesto por 10 ítems expresados en una escala Likert de 5 puntos que va de 0 (totalmente en desacuerdo) a 4 (totalmente de acuerdo). Del total de los ítems, cuatro son distractores, tres aluden a una visión optimista y tres a una visión pesimista. La adaptación española mostró una estimación de la confiabilidad de .7 y en la adaptación en muestra chilena fue de .65 (Chico, 2002; Ferrando, Chico \& Tous, 2002; Vera-Villarroel, Córdoba-Rubio, \& Celis-Atenas, 2009) 
En este estudio solo se utilizó la escala de optimismo (Alfa de Cronbach de .726) para determinar la mediación de la variable en la relación entre ansiedad como rasgo y las diferentes dimensiones del bienestar psicológico.

\section{Procedimientos}

El presente proyecto de investigación fue revisado y aprobado por el Comité Ético Científico de la Universidad Católica de Norte. Antes de aplicar los instrumentos, a los estudiantes se les daba a conocer el objetivo de la investigación, dejando claro que su participación es totalmente voluntaria. En todo el proceso investigativo se ha resguardado la confidencialidad y el anonimato de los estudiantes encuestados.

\section{Análisis de datos}

Para evaluar la normalidad se aplicó la Prueba de normalidad de KolmogorovSmirnof con la corrección de la significación de Lilliefors (Pedrosa, Juarros-Basterretxea, Robles-Fernández, Basteiro, \& García-Cueto, 2014), la cual entregó los siguientes resultados: Optimismo $[\mathrm{K}-\mathrm{S}(332)=.134, p=.0001]$, Ansiedad como Rasgo [K-S(332) $=.067, p=.001]$, Autoaceptación $[\mathrm{K}-\mathrm{S}(332)=.105, p=.0001]$, Relaciones positivas [K$\mathrm{S}(332)=.095, p=.0001]$, Autonomía [K-S $(332)=.073, p=.0001]$, Dominio del entorno [K$\mathrm{S}(332)=.055, p=.016]$, Crecimiento personal [K-S $(332)=.101, p=.0001]$, Propósito en la vida $[\mathrm{K}-\mathrm{S}(332)=.113, p=.0001]$. En todos los casos se rechaza la hipótesis nula $\left(\mathrm{H}_{0}\right)$, no cumpliéndose el supuesto de normalidad.

Dado el tamaño de la muestra $(\mathrm{N}=332)$ se utilizaron pruebas paramétricas, las cuales han demostrado ser robustas cuando se violan tanto el supuesto de normalidad como el de homocedasticidad (Finch, 2005; Lemeshko \& Lemeshko, 2008). Para los análisis descriptivos y de correlación se utilizó el programa estadístico SPSS v.21 y para los análisis de mediación se utilizó el software PROCESS for SPSS de Andrew F. Hayes.

\section{Resultados}

A continuación, en la Tabla 1 se presentan estadísticos descriptivos para las variables de estudio. En la Tabla 2 se presentan las correlaciones obtenidas entre las variables de estudio. Estos resultados muestran correlaciones significativas entre todas las variables, donde la Ansiedad como Rasgo correlaciona en forma negativa y el Optimismo en forma positiva con todas las dimensiones del BP. 
Tabla 1.

Media y desviación estándar para variables de estudio

\begin{tabular}{lll}
\hline & Media & Desv. típ. \\
\hline Ansiedad como rasgo & 1,9519 &, 45053 \\
Optimismo & 3,8439 &, 80790 \\
Autoaceptación & 4,5776 &, 83980 \\
Relaciones positivas & 4,5795 &, 97066 \\
Autonomía & 4,3239 &, 87714 \\
Dominio del entorno & 4,3518 &, 82225 \\
Crecimiento Personal & 4,9157 &, 83248 \\
Propósito en la vida & 4,6392 &, 90051 \\
\hline
\end{tabular}

Tabla 2

Correlación de Pearson entre variables de estudio

\begin{tabular}{llllllll}
\hline \multicolumn{1}{c}{ AR } & OP & AABP & RPBP & ABP & DEBP & CPBP \\
\hline OP &,$- 373^{* *}$ & & & & & & \\
AABP &,$- 456^{* *}$ &, $601^{* *}$ & & & & & \\
RPBP &,$- 196^{* *}$ &, $217^{* *}$ &, $405^{* *}$ & & & & \\
ABP &,$- 305^{* *}$ &, $203^{* *}$ &, $488^{* *}$ &, $326^{* *}$ & & & \\
DEBP &,$- 388^{* *}$ &, $419^{* *}$ &, $664^{* *}$ &, $371^{* *}$ &, $443^{* *}$ & & \\
CPBP &,$- 193^{* *}$ &, $455^{* *}$ &, $569^{* *}$ &, $372^{* *}$ &, $355^{* *}$ &, $562^{* *}$ & \\
PVBP &,$- 358^{* *}$ &, $606^{* *}$ &, $753^{* *}$ &, $358^{* *}$ &, $359^{* *}$ &, $742^{* *}$ &, $606^{* *}$ \\
\hline
\end{tabular}

* La correlación es significativa al nivel 0,01 (bilateral).

**AABP $=$ autoaceptación $; \mathrm{RPBP}=$ relaciones positivas con los otros; $\mathrm{ABP}=$ autonomía $; \mathrm{DEBP}=\mathrm{Dominio}$ del entorno; $\mathrm{CPBP}=$ crecimiento personal; $\mathrm{PVBP}=$ propósito en la vida.

\section{Modelos de mediación simple.}

Se utilizaron modelos de mediación simple para evaluar el efecto mediador del optimismo en la relación AR y las diferentes dimensiones del BP como variables dependientes. Los análisis de regresión indicaron que la variable independiente AR, se asoció significativamente con la variable mediadora $\mathrm{OP}(B=-0,67, E T=.092, t=-7,30$, $p<0.001,, 95 \%$ CI [-0.8502, -0,4893]). Al evaluar el efecto de la AR, mediado por el OP, sobre las diferentes dimensiones del BP, se obtuvieron los siguientes resultados, los cuales pueden ser visualizados en las Figura 1 y 2.

\section{Optimismo como mediador entre la ansiedad como rasgo y autoaceptación.}

En el caso de la dimensión de autoaceptación del BP (variable dependiente), esta se asoció significativa y positivamente con la variable mediadora de OP $(B=0.52, T E=$ 0.0469, $t=11.08, p<0.001,95 \%$ CI [0.4276, 0.6122]). En este modelo, la AR (variable independiente) se asoció significativa y negativamente con la variable autoaceptación 
$(B=-0.85, T E=0.0913, t=-9.30, p<0,001,95 \%$ CI $[-1.0297,-0.6704])$ pero su efecto se vio reducido con la incorporación de la variable optimismo en la regresión $(B=-0.50$, $T E=0.0841, t=-5.96, p<0.001,95 \%$ CI [-0.6675, -0.3365]) (ver Figura 1a). Asimismo, la prueba de efecto indirecto basada en el procedimiento bootstrap fue significativa para el efecto indirecto a través del optimismo ( $B=-0.1867$, Boot $E T=0.02,95 \%$ CI [-0.2466, $-0.1311])$.

Optimismo como mediador entre la ansiedad como rasgo y relaciones positivas con los otros.

En el caso de la dimensión de relaciones positivas con los otros del BP, esta se asoció significativa y positivamente con la variable mediadora de OP $(B=0.23, T E=$ 0.0726, $t=3.17, p<0.01,95 \%$ CI $[0.0880,0.3737)$. En este modelo, la AR se asoció significativa y negativamente con la variable relaciones positivas con los otros $(B=-0.45$, $T E=0.1224, t=-3.69, p<0.001,95 \%$ CI $[-0.6926,-0.2109])$, y su efecto se vio reducido con la incorporación de la variable optimismo en la regresión $(B=-0.29, T E=0.1302, t=-$ $2.28, p<0,05,95 \%$ CI $[-0.5533,-0.0411]$ ) (ver Figura 1b). La prueba de efecto indirecto basada en el procedimiento bootstrap fue significativa para el efecto indirecto a través del optimismo $(B=-0.0681$, Boot $E T=0.02,95 \%$ CI [-0.1214, -0.0337]).

\section{Optimismo como mediador entre la ansiedad como rasgo y autonomía.}

En tanto la variable autonomía del BP se asoció significativa y positivamente con la variable mediadora OP $(B=0.14, T E=0.0636, t=-2.17, p<0.05,95 \%$ CI $[0.0135$, 0.2639). En este modelo, la AR se asoció significativa y negativamente con la variable autonomía $(B=-0.59, T E=0.1065, t=-5.61, p<0,001,95 \%$ CI $[-0.8070,-0.3881])$ y al igual que en los modelos anteriores su efecto se vio reducido con la incorporación de la variable optimismo en la regresión $(B=-0.50, T E=0.1141, t=-4.42, p<0.001,95 \%$ CI [0.7292, -0.2802]) (ver Figura 1c). En tanto, la prueba de efecto indirecto basada en el procedimiento bootstrap fue significativa para el efecto indirecto a través del optimismo $(B=0.0459$, Boot $E T=0.02,95 \%$ CI $[-0.0960,-0.0002])$. 

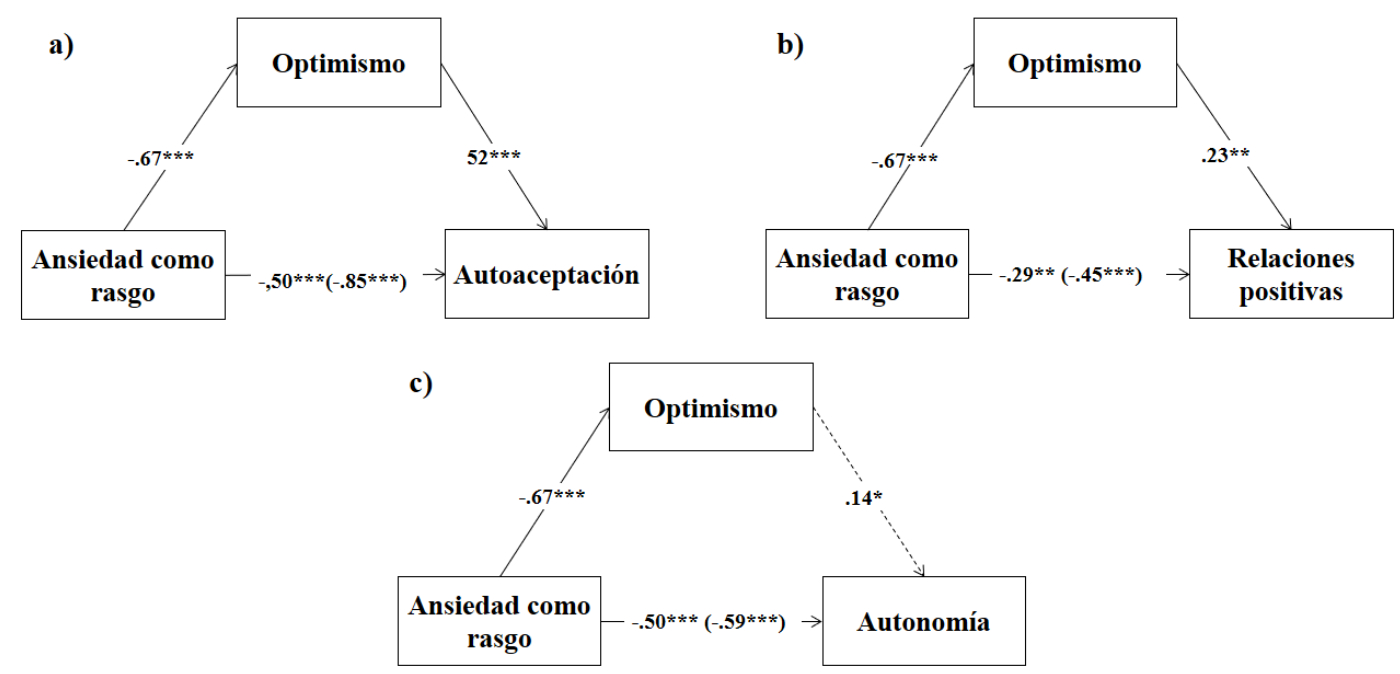

Figura 1. Optimismo como mediador parcial entre la ansiedad como rasgo y las dimensiones de autoaceptación, relaciones positivas con los otros y autonomía del bienestar psicológico.

Fuente: elaboración propia.

\section{Optimismo como mediador entre la ansiedad como rasgo y dominio del entorno.}

En el caso de la dimensión de dominio del entorno del BP, esta se asoció significativa y positivamente con la variable mediadora $\mathrm{OP}(B=0.36, T E=0.0547, t=6.54$, $p<0.001,95 \%$ CI [0.2506, 0.4659]). En este modelo, la AR se asoció significativa y negativamente con la variable dominio del entorno $(B=-0.72, T E=0.0966, t=-7.47, p$ $<0,001,95 \%$ CI [-0.9120, -0.5319]), pero su efecto se vio reducido con la incorporación de la variable optimismo en la regresión $(B=-0.48, T E=0.0981, t=-4,91, p<0,001,95 \%$ [-0.6751, -0.2891]) (ver Figura 2a). Asimismo, la prueba de efecto indirecto basada en el procedimiento bootstrap fue significativa para el efecto indirecto a través del optimismo $(B=-0.1264$, Boot $E T=0.02,95 \%$ CI $[-0.1868,-0.0799])$.

\section{Optimismo como mediador entre la ansiedad como rasgo y crecimiento personal.}

En el caso de la variable de crecimiento personal del BP (variable dependiente), esta se asoció significativa y positivamente con la variable mediadora $\mathrm{OP}$ ( $B=0.47, T E=$ $0.0561, t=8,43 ; p<0,001,95 \%$ [ 0.3628, 0.5834]). En este modelo, la AR se asoció significativa y negativamente con la variable crecimiento personal $(B=-0.37, T E=0.1027$, $t=-3,64, p<0,001,95 \%[-0,5769,-0.1728])$, y su efecto se vio reducido hasta no ser significativo con la incorporación de la variable optimismo en la regresión $(B=-0,058$, $T E=0.1005, t=-0,58, \quad p>0,5,95 \% \quad[-0.2559,-0.1397])$, produciéndose una mediación total del efecto de AR sobre la variable crecimiento personal (ver Figura 2b). La prueba 
de efecto indirecto basada en el procedimiento bootstrap fue significativa para el efecto indirecto a través del optimismo $(B=-0.1664$, Boot $E T=0.02,95 \%$ CI $[-0.2269$, $0.1155])$.

Optimismo como mediador entre la ansiedad como rasgo y propósito de vida.

En el caso de la variable de propósito de vida del BP, esta se asoció significativa y positivamente con la variable mediadora $\mathrm{OP}(B=0.61, T E=0.0518, t=11.80, p<0.001$, 95\% CI [0.05098, 0.7138]), En este modelo, la AR (variable independiente) se asoció significativa y negativamente con la variable propósito de vida $(B=-0.71, T E=0.1027$, $t=-6.96, p<0,001,95 \%$ CI [-0.9177, -0.5135), y al igual en todos los casos anteriores, su efecto se vio disminuido con la incorporación de la variables OP $(B=-0.30, T E=0.0930$, $t=-3.29, p<0,01,95 \%$ CI $[-0.4889,-0.1231]$ ) (ver Figura 2c). En tanto, la prueba de efecto indirecto basada en el procedimiento bootstrap fue significativa para el efecto indirecto a través del optimismo $(B=-0.2049$, Boot $E T=0.03,95 \%$ CI [-0.2763, $0.1439])$.
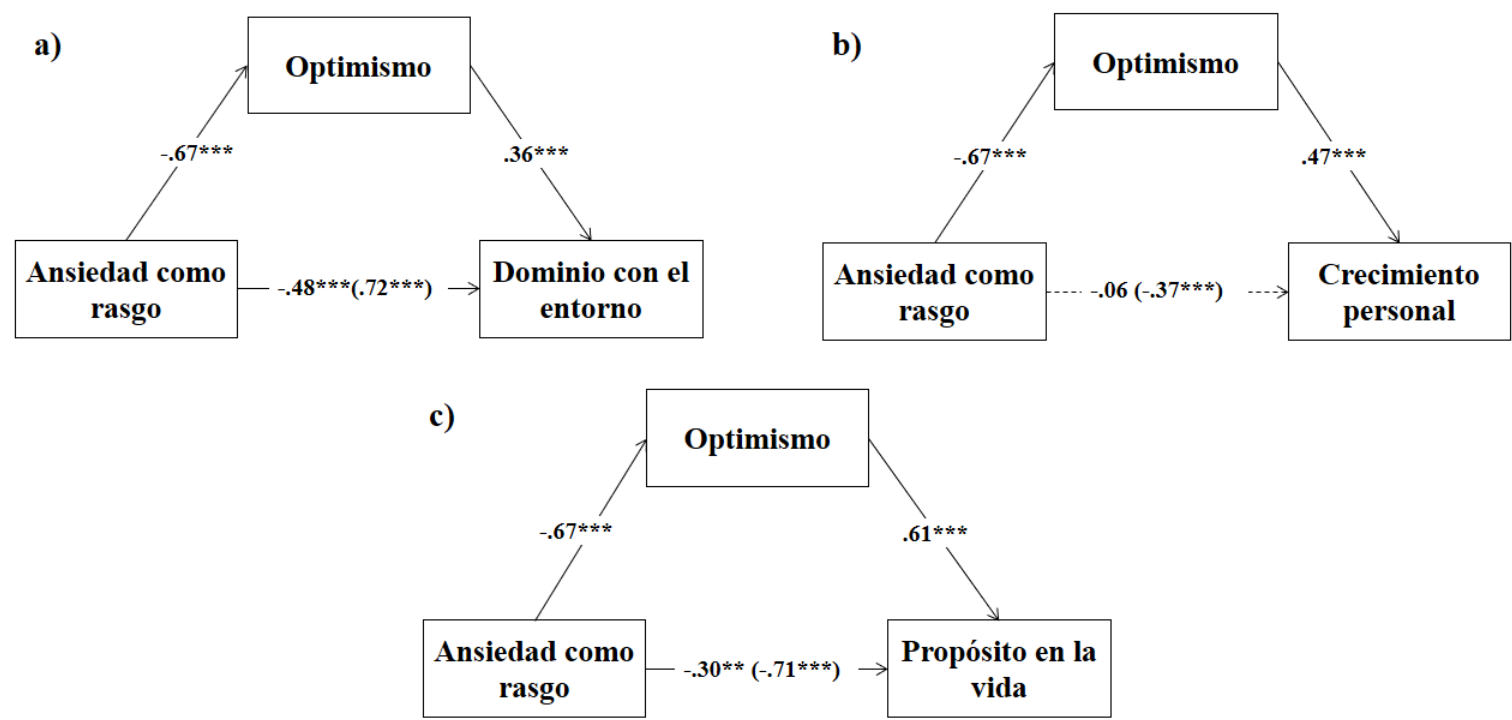

Figura 2. Optimismo como mediador parcial y total entre la ansiedad como rasgo y las dimensiones de dominio del entorno, crecimiento personal y propósito en la vida del bienestar psicológico.

Fuente: elaboración propia.

En resumen, el OP explica parcialmente la relación entre AR y las diferentes dimensiones del BP, con excepción de la dimensión crecimiento personal, donde se produce una mediación total en el efecto de la AR sobre esta variable. 


\section{Discusión}

El objetivo de este estudio fue evaluar el rol mediador del OP en la relación entre AR y las diferentes dimensiones del BP. Los hallazgos de este estudio concuerdan con los resultados obtenidos en estudios previos, que muestran una relación significativa entre AR y el BP (Gutiérrez, 2017; Pardo, 2010; Taoka et al., 2014; Ruini et al., 2003; Shek, 1993; Villaseñor-Ponce, 2010), AR y OP (Pavez, Mena, et al., 2012), OP y BP (Augusto-Landa et al., 2011; Ferguson \& Goodwin, 2010; Vera-Villarroel, et al., 2009) y el modo en que el optimismo juega un papel mediador en esta relación.

La posibilidad de que el optimismo medie la relación AR-BP podría ser explicada basándose en la teoría de autoregulación (Carver \& Scheir, 1990), la cual propone que las personas se esfuerzan en la medida que perciben como alcanzables sus objetivos, logrando así, experimentar resultados que inciden positivamente en su autorealización. En este caso, los efectos negativos de la AR - que influyen positivamente en la percepción de amenazas - son mediados por el efecto del optimismo, el cual se relaciona positivamente con una mayor percepción de autoeficacia y control sobre los resultados esperados (Gillham, Shatte, Reivich, \& Seligman, 2001), lo cual se asocia a mayores niveles de BP.

Los resultados de este estudio dan cuenta del importante efecto negativo de la AR sobre las diferentes dimensiones del BP de los estudiantes, efecto que se ve disminuido al considerar el OP. Los estudiantes con este rasgo de personalidad (AR) presentan mayores niveles de ansiedad al experimentar una mayor percepción de amenazas frente a las exigencias propias de la vida universitaria, lo cual afecta en forma negativa su bienestar psicológico. Frente a esta situación, el fortalecimiento del optimismo puede ser visto como una estrategia para disminuir los efectos negativos de la AR en el estudiante universitario.

Desde este punto de vista, los hallazgos de este estudio evidencian la importancia de implementar en la educación terciaría, programas u otras estrategias de desarrollo de habilidades y competencias que fortalezcan los recursos psicológicos de los estudiantes. En este caso, el optimismo fortalecería el bienestar psicológico, actuando como factor protector frente a las amenazas percibidas durante la formación universitaria, y en un futuro, como un factor que influye positivamente en la adaptación y en el desenvolvimiento en al ámbito laboral. 
Si bien los resultados de este estudio son preliminares, aportan con evidencia a los modelos Top Down (Castro, 2009), explicando los niveles de bienestar desde la influencia de variables internas (Mc Nulty \& Finchman, 2012). Creemos que es importante evaluar la estabilidad de las variables estudiadas y aumentar la validez interna del estudio, por lo cual es necesario replicar este modelo en contextos longitudinales y/o a un nivel experimental, con lo cual se contribuiría, con mayor evidencia teórica y práctica, al desarrollo de estrategias que fortalecen el bienestar psicológico de los estudiantes universitarios. 


\section{Referencias}

Abdel-Khalek, A. (2006). Measuring happiness with a single-item scale. Social Behavior and Personality, 34(2), 139-150. https://doi.org/10.2224/sbp.2006.34.2.139

Agudelo, D., Casadiegos, C. \& Sánchez, D. (2008). Características de ansiedad y depresión en estudiantes universitarios. International Journal of Psychological Research, 1(1), 3439.

Allport, G.W. (1961). Pattern and growth in personality. Holt, Rinehart, \& Winston.

Augusto-Landa, J.M., Pulido-Martos, M. \& López-Zafra, E. (2011). Does Perceived Emotional Intelligence and Optimism/pessimism Predict Psychological Wellbeing? Journal Happiness Studies, 12(3), 463-474. https://doi.org/10.1007/s10902-010-9209$\underline{7}$

Bodas, J., Ollendick, T. H. y Sovani, A. (2008). Test anxiety in Indian children: A crosscultural perspective. Anxiety, Stress \& Coping, 21(4), 387-404. https://doi.org/10.1080/10615800701849902

Brydon, L., Walker, C., Wawrzyniak, A. J., Chart, H. \& Syeptoc, A. (2009). Dispositional optimism and stress-induced changes in immunity and negative mood. Behavioral and Inmunity, 23(6), 810-816. https://doi.org/10.1016/j.bbi.2009.02.018

Cardona-Arias, J.A., Pérez-Restrepo, D., Rivera-Ocampo, S., Gómez-Martínez, J., \& Reyes, A. (2015). Prevalencia de ansiedad en estudiantes universitarios. Diversitas: Perspectivas en Psicología, 11(1), 79-89. https://doi.org/10.15332/s17949998.2015.0001.05

Carmona-Halty, M. \& Rojas-Paz, P. (2014). Rasgos de personalidad, necesidades de cognición y satisfacción vital en estudiantes universitarios chilenos. Universitas Psychologica, 13(1), 83-93. https://revistas.javeriana.edu.co/index.php/revPsycho/article/view/1757

Castro, A. (2009). El bienestar psicológico: cuatro décadas de progreso. Revista Interuniversitaria de Formación del Profesorado, 23(3), 43-72.

Casullo, M. M. (2002). Evaluación del bienestar psicológico en Iberoamérica. Paidós.

Carver, C. S., \& Scheier, M. (1990). Principles of selfregulation: Action and emotion. En T. E. Higgins \& R. M. Sorrentino (Eds.), Handbook of motivation and cognition: Foundations of social behavior (pp. 3-52). Guilford Press.

Chávez, A. (2006). Bienestar psicológico y su influencia en el rendimiento académico de estudiantes de nivel medio superior [Tesis de maestría]. Universidad de Colima, México.

Chico, E. (2002). Optimismo disposicional como predictor de estrategias de afrontamiento. Psicothema, 14(3), 544-550.

Chitgian-Urzúa, V., Urzúa, A., \& Vera-Villarroel, P. (2013). Análisis preliminar de las Escalas de Bienestar Psicológico en población chilena. Revista Argentina de Clínica Psicológica, 22(1), 5-14.

Díaz, D., Rodríguez, R., Blanco, A., Moreno, B., Gallardo, I., Valle, C. \& van Dierendonck, D. (2006). Adaptación española de las Escalas de Bienestar Psicológico de Ryff. Psicothema, 18(3), 572-577.

D’Anello, S. (2006). Efecto de las normas y emociones en los juicios sobre satisfacción con la vida en una muestra venezolana. Revista Interamericana de psicología, 40(3), 371376.

De Moor, J. S., De Moor, C. A., Basen-Engquist, K., Kudelka, A., Bevers, M. W. \& Cohen, L. (2006). Optimism, distress, health-related quality of life, and change in cancer antigen 125 among patients with ovarian cancer undergoing chemotherapy. 
Psychosomatic Medicine, 68(4), 555-562.

https://doi.org/10.1097/01.psy.0000222379.71389.91

DeNeve, K. \& Cooper, H. (1998). The happy personality: a meta-analysis of 137 personality traits and subjective wellbeing. Psychological Bulletin, 124(2), 197-229. doi: 10.1037/0033-2909.124.2.197

Eum, K. \& Rice, K. G. (2011). Test anxiety, perfectionism, goal orientation, and academic performance. Anxiety, Stress, and Coping, 24(2), 167-178.

https://doi.org/10.1080/10615806.2010.488723

Ferguson, S. \& Googwin, A. (2010). Optimism and Well-Being in Older Adults: The Mediating Role of Social Support and Perceived Control. The International Journal of Aging and Human Development, 71(1), 43-68. https://doi.org/10.2190/AG.71.1.c

Finch, H. (2005). Comparison of the performance of nonparametric and parametric MANOVA test statistics when assumptions are violated. Methodology, 1(1), 27-38. https://doi.org/10.1027/1614-1881.1.1.27

Ferrando, P.J., Chico, E. \& Tous, J.M. (2002). Propiedades psicométricas del test de optimismo Life Orientation Test. Psicothema, 14(3), 673-680.

Freire, C. (2014). El bienestar psicológico en los estudiantes universitarios: operativización del constructo y análisis de su relación con las estrategias de afrontamiento [Tesis doctoral]. Universidade da Coruña, España.

Freire, C., Ferradás, M., Núñez, J., \& Valle, A. (2017). Estructura factorial de las Escalas de Bienestar Psicológico de Ryff en estudiantes universitarios. European Journal of Education and Psychology, 10(1), 1-8. https://doi.org/10.1016/j.ejeps.2016.10.001

García-Alandete, Joaquín. (2013). Bienestar psicológico, edad y género en universitarios españoles. Salud \& Sociedad: investigaciones en psicología de la salud y psicología social, 4(1), 48-58.

Gillham, J.E., Shatté, A.J., Reivich, K.J. \& Seligman, M.E.P. (2001). Optimism, pessimism and explanatory style. En E.C. Chang (ed.): Optimism and pessimism. Implications for theory, research and practice (pp. 53-75). American Psychological Association.

Grant, S., Langan-Fox, J. \& Anglim, J. (2009). The big five traits as predictors of subjetive and psychological well-being. Psychological Reports, 105(1), 205-231. https://doi.org/10.2466/PR0.105.1.205-231

Gutiérrez, M. (2017). Bienestar psicológico y ansiedad en estudiantes de una universidad nacional del norte del Perú. [Tesis de licenciatura]. Universidad Privada del Norte, Perú.

Kivimaki, M., Elovainio, M., Singh-Manoux, A., Vahtera, J., Helenius, H. \& Pentti, J. (2005). Optimism and pessimism as predictors of change in health after death or onset of severe illness in family. Health Psychology, 24(4), 413-421. https://doi.org/10.1037/0278-6133.24.4.413

Lemeshko, B., \& Lemeshko, S. (2008). Power and robustness of criteria used to verify the homogeneity of means. Measurement Techniques, 51(9), 950-959. https://doi.org/10.1007/s11018-008-9157-3

Maslow, A. H. (1968). Toward a psychology of being. D. Van Norstrand.

McNulty, J., \& Fichman, F. (2012). Beyond Positive Psychology? Toward a Contextual View of Psychological Processes and Well-Being. American Psychologist, 67(2), 101-110. https://doi.org/10.1037/a0024572

Molina, C., \& Meléndez, J. (2006). Bienestar psicológico en envejecientes de la República Dominicana. Geriátrika, 22(3), 97-105.

Muratori, M. Z., \& Bobowik, J. L. (2015). Felicidad y Bienestar Subjetivo: Estudio Comparativo entre Argentina y España. Psykhe, 24(2), 1-18. https://doi.org/10.7764/psykhe.24.2.900 
Pardo, F. (2010). Bienestar psicológico y ansiedad rasgo-estado en alumnos de un MBA de Lima Metropolitana. [Tesis de licenciatura]. Pontifica Universidad Católica del Perú.

Pavez, P., Mena, L. \& Vera-Villarroel, P. (2012). El rol de la felicidad y el optimismo como factor protector de la ansiedad. Universitas Psychologica, 11(2), 369-380.

Pedrosa, I., Juarros-Basterretxea, J., Robles-Fernández, A., Basteiro, J. \& García-Cueto, E. (2015). Pruebas de bondad de ajuste en distribuciones simétricas, ¿qué estadístico utilizar? Universitas Psychologica, 14(1), 245-254.

https://dx.doi.org/10.11144/Javeriana.upsy13-5.pbad

Perez. J.A. (2012). Gender difference in psychological well-being among Filipino college student samples. International Journal of Humanities and Social Science, 2(13) 84-93.

Rogers, C.R. (1961). On becoming a person. Houghton Mifflin.

Ruini, C., Ottolini, F., Rafanelli, C., Tossani, E., Ryff, C. D. \& Fava, G. A. (2003). The relationship of psychological well-being to distress and personality. Psychotherapy and Psychosomatics, 72(5), 268-275. https://dx.doi.org/10.1159/000071898

Ryan, R., \& Deci, E. (2001). On happiness and human potentials: A review of research on hedonic and eudaimonic well-being. Annual Review of Psychology, 52, 141-166. https://dx.doi.org/10.1146/annurev.psych.52.1.141

Ryff, C. D. (1989). Happiness is everything, or is it? Explorations on the meaning of psychological well-being. Journal of Personality and Social Psychology, 57(6), 10691081. https://dx.doi.org/10.1037/0022-3514.57.6.1069

Ryff, C. D. \& Keyes, C. L. M. (1995). The structure of psychological well-being revisited. Journal of Personality and Social Psychology, 69(4), 719-727. https://dx.doi.org/10.1037/0022-3514.69.4.719

Ryff, C. \& Singer, B. (1998). The contours of positive health. Psychological Inquiry, 9(1), 128. https://dx.doi.org/10.1207/s15327965pli0901_1

Sandoval, S., Dorner, A., \& Véliz, A. (2017). Bienestar psicológico en estudiantes de carreras de la salud. Investigación en Educación Médica, 6(24), 260-266. https://dx.doi.org/10.1016/j.riem.2017.01.004

Schimmack, U., Radhakrishnan, P., Oishi, S., Dzokoto, V., \& Ahadi, S. (2002). Culture, personality, and subjective well-being: Integrating process models of life satisfaction. Journal of Personality and Social Psychology, 82(4), 582-593. https://dx.doi.org/10.1037/0022-3514.82.4.582

Schmutte, P.S. \& Ryff, C.D. (1997). Personality and well-being: Reexamining methods and meanings. Journal of Personality and Social Psychology, 73(3), 549-559. https://dx.doi.org/10.1037/0022-3514.73.3.549

Shek, D. T. (1993). The Chinese version of the State-Trait Anxiety Inventory: Its relationship to different measures of psychological well-being. Journal of Clinical Psychology, 49(3), 349-358. https://doi.org/10.1002/1097-4679(199305)49:3<349::AIDJCLP2270490308>3.0.CO;2-J

Spielberger, C.D., Gorsuch, R., \& Lushene, R. (1970). Manual for the StateTrait Anxiety Inventory. Consulting Psychologist Press.

Spielberger, C. D. \& Díaz-Guerrero, R. (1975). IDARE Inventario de Ansiedad: RasgoEstado. El Manual Moderno.

Spielberger, C. D. (1989). State-Trait Anxiety Inventory: A comprehensive bibliography. Consulting Psychologists Press.

Steel, P., Schmidt, J., \& Shultz, J. (2008). Refining the relationship between personality and subjective well-being. Psychological Bulletin, 134(1), 138-161. https://doi.org/10.1037/0033-2909.134.1.138

Scheier, M.F., Carver, C.S. \& Bridges, M.W. (1994). Distinguising optimism from neuroticism (and trait anxiety, self mastery and self esteem): A reevaluation of the Life 
Orientation Test. Journal of Personality and Social Psychology, 67(6), 1063-1078. https://doi.org/10.1037/0022-3514.67.6.1063

Taoka, R., Matsunaga, H., Kubo, T., Suzuki, T., \& Yamamoto, S. (2014). Impact of trait anxiety on psychological well-being in men with prostate cancer. International braz $j$ urol, 40(5), 620-626. https://doi.org/10.1590/S1677-5538.IBJU.2014.05.06

Tirado, J., Ortega, S., Díaz, V. \& Martín, F. (2005). Terapia breve en estudiantes universitarios con problemas de rendimiento académico y ansiedad: Eficacia del modelo 'La cartuja'. International Journal of Clinical and Health Psychology, 5(3), 589-608.

Triandis, H. (1994). Culture and social behavior. McGraw-Hill.

van Dierendonck, D. (2004). The construct validity of Ryff's Scale of Psychological wellbeing and its extension with spiritual well-being. Personality and Individual Differences, 36(3), 629-644. https://doi.org/10.1016/S0191-8869(03)00122-3

Velásquez, C., Montgomery, W., Montero, V., Pomalaya, R., Dioses, A., Velásquez, N., Araki, R., \& Reynoso, D. (2008). Bienestar psicológico, asertividad y rendimiento académico en estudiantes universitarios sanmarquinos. Revista de Investigación en Psicología, 11(2), 139-152. https://doi.org/10.15381/rinvp.v11i2.3845

Veliz-Burgos, A., \& Apodaca, P. (2012). Niveles de autoconcepto, autoeficacia académica y bienestar psicológico en estudiantes universitarios de la ciudad de Temuco. Salud \& Sociedad, 3(2), 131-150.

Véliz-Burgos, A. (2012). Propiedades psicométricas de la escala de bienestar psicológico y su estructura factorial en universitarios chilenos. Psicoperspectivas: Individuo y Sociedad, 11(2), 143-163. https://doi.org/10.5027/psicoperspectivas-Vol11-Issue2$\underline{\text { fulltext-196 }}$

Vera, B. (2006). Psicología positiva: una nueva forma de entender la Psicología. Papeles del Psicólogo, 27(1), 3-8.

Vera-Villarroel, P., Córdova-Rubio, N., \& Celis-Atenas, K. (2009). Optimismo versus autoestima: implicancia para la psicología clínica y la psicoterapia. Revista Argentina de Clínica Psicológica, 18(1), 21-30.

Vera-Villarroel, P., Urzúa, A., Silva, J., Pavez, P. \& Celis-Atenas, K. (2013). Escala de Bienestar de Ryff: Análisis Comparativo de los Modelos Teóricos en Distintos Grupos de Edad. Psicologia: Reflexão e Crítica, 26(1), 106-112.

Vera-Villarroel, P., Celis-Atenas, K., Córdova-Rubio, N., Buela-Casal, G. \& Spielberger, C. D. (2007). Preliminary analysis and normative data of the State-Trait Anxiety Inventory (STAI) in adolescent and adults of Santiago, Chile. Terapia Psicológica, 25(2), 155-162. https://doi.org/10.4067/S0718-48082007000200006

Vera-Villarroel, P., Pavez, P., \& Silva, J. (2012). El rol predisponente del optimismo: hacia un modelo etiológico del bienestar. Terapia Psicológica, 30(2), 77-84. https://doi.org/10.4067/S0718-48082012000200008

Villarroel, V., \& Bruna, D. (2014). Reflexiones en torno a las competencias genéricas en educación superior: Un desafío pendiente. Psicoperspectivas, 13(1), 2234. https://doi.org/10.5027/psicoperspectivas-Vol13-Issue1-fulltext-335

Villaseñor-Ponce, M. (2010). Correlación entre la ansiedad y bienestar psicológico en estudiantes que ingresan a la universidad. Revista mexicana de psicología educativa, 1(1), 41-48.

Weiss, A., Bates, T. C. \& Luciano, M. (2008). Happiness Is a Personal(ity) Thing The Genetics of Personality and Well-Being in a Representative Sample. Psychological Science, 19(3), 205-210. https://doi.org/10.1111/j.1467-9280.2008.02068.x

Zubieta, E., Muratori, M. \& Fernández, O. (2012). Bienestar subjetivo y psicosocial: explorando diferencias de género. Salud \& Sociedad, 3(1), 66-76. 